\title{
Distance Coordinates With Respect to a Triangle of Reference
}

\section{Karl Goldberg}

\author{
Institute for Basic Standards, National Bureau of Standards, Washington, D.C. 20234
}

(July 21, 1972)

\begin{abstract}
With respect to a triangle of reference $A_{1} A_{2} A_{3}$, each point $P$ in the plane of the triangle, has unique area coordinates: $P=\left(b_{1}, b_{2}, b_{3}\right)$ with $b_{1}+b_{2}+b_{3}=1$. Distance coordinates are introduced such that $P=\left[d_{1}, d_{2}, d_{3}\right]$, with $d_{k}$ the distance from $P$ to $A_{k}$. It is shown that there is an explicit function $f\left(x_{1}, x_{2}, x_{3}\right)$ such that $f\left(d_{1}^{2}, d_{2}^{2}, d_{3}^{2}\right)=0$ is necessary and sufficient for $P=\left[d_{1}, d_{2}, d_{3}\right]$, each $d_{k}$ nonnegative. The partial derivatives $f_{k}\left(x_{1}, x_{2}, x_{3}\right)=\partial f\left(x_{1}, x_{2}, x_{3}\right) / \partial x_{k}$ are such that $b_{k}=f_{k}\left(d_{1}^{2}\right.$, $\left.d_{2}^{2,} d_{3}^{2}\right)$ for each $k$. Other results relating the $b_{k}$ and the $d_{k}$ are given. The use of $f\left(x_{1}, x_{2}, x_{3}\right)$ in solving geometric problems is shown.
\end{abstract}

Key words: Area coordinates; distance coordinates; Plane Geometry; radical center; triangle of reference.

We are given three noncollinear points $A_{1}, A_{2}, A_{3}$ and all other points are in the plane of the triangle of reference $A_{1} A_{2} A_{3}$.

Notationally, two distinct points $X, Y$ determine an infinite line $X Y$, with the finite line segment $\overline{X Y}$ having length $|X, Y|$. If $X, Y$ are centers of circles with radii $x, y$ respectively, the radical axis of those circles is a line perpendicular to $X Y$, at a point which is $\left(|X, Y|^{2}+x^{2}-y^{2}\right) / 2|X, Y|$ from $X$ in the direction of $Y$. Given a third point $Z$, not on $X Y$, as the center of a circle, the three radical axes meet in a common point called their radical center. The area of the triangle $X Y Z$ is denoted by $|X, Y, Z|$. The function

$$
F\left(x_{1}, x_{2}, x_{3}\right)=2\left(x_{1} x_{2}+x_{1} x_{3}+x_{2} x_{3}\right)-x_{1}^{2}-x_{2}^{2}-x_{3}^{2}
$$

has the well-known property

$$
16|X, Y, Z|^{2}=F\left(|X, Y|^{2},|X, Z|^{2},|Y, Z|^{2}\right) .
$$

Let $\Delta=\left|A_{1}, A_{2}, A_{3}\right|$ denote the area of the triangle of reference. With respect to this triangle every point $P$ has unique area coordinates ${ }^{1} b_{1}, b_{2}, b_{3}$, which are real numbers restricted by

$$
b_{1}+b_{2}+b_{3}=1
$$

We write

$$
P=\left(b_{1}, b_{2}, b_{3}\right)
$$

with $A_{1}=(1,0,0), A_{2}=(0,1,0), A_{3}=(0,0,1)$.

AMS Subject Classification: Primary 5010.

'Also called "normalized barycentric" or "areal" coordinates [1]. ${ }^{2}$

${ }^{2}$ Coxeter, H.S.M., Introduction to Geometry (Wiley, 1961). 
The area coordinate $b_{1}$ is defined as the ratio $\pm\left|P, A_{2}, A_{3}\right| / \Delta$ with $b_{1}>0$ if $\overline{A_{1} P}$ does not intersect $A_{2} A_{3}, b_{1} \leqslant 0$ if it does. Similarly for the other $b_{k}$, so that the diagram of signs is

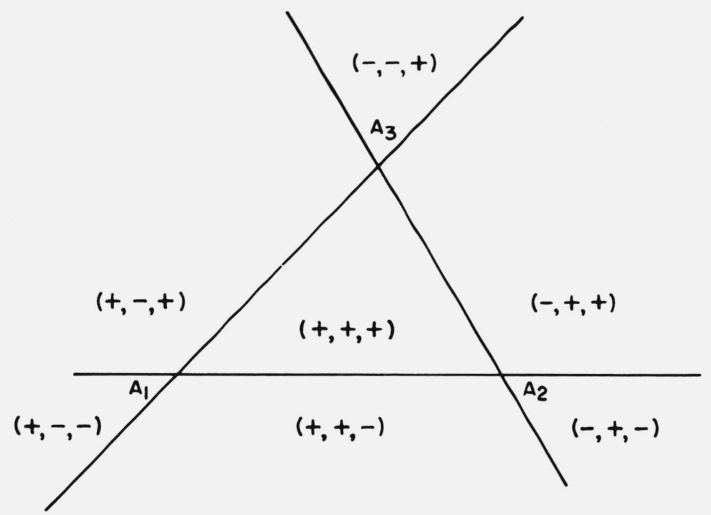

Conversely, any three real numbers $b_{1}, b_{2}, b_{3}$, restricted by $(3)$, define a unique point $P=\left(b_{1}, b_{2}\right.$, $b_{3}$ ) with respect to $A_{1} A_{2} A_{3}$.

If a point $P$ is at a distance $d_{1}$ from $A_{1}, d_{2}$ from $A_{2}$, and $d_{3}$ from $A_{3}$, we call these the distance coordinates of $P$ with respect to $A_{1} A_{2} A_{3}$, and write

$$
P=\left[d_{1}, d_{2}, d_{3}\right]
$$

or, when more convenient,

$$
P=<d_{1}^{2}, d_{2}^{2}, d_{3}^{2}>\text {. }
$$

For clarity, $d_{k}=\left|P, A_{k}\right|$ for all $k=1,2,3$.

Note that the triangle $P A_{2} A_{3}$ has side lengths $a_{1}, d_{2}, d_{3}$ so that

$$
16 \Delta^{2} b_{1}^{2}=F\left(a_{1}^{2}, d_{2}^{2}, d_{3}^{2}\right)
$$

with similar equations for the other $b_{k}$. Also note that $P$ is the radical center of circles with centers $A_{1}, A_{2}, A_{3}$ and radii $d_{1}, d_{2}, d_{3}$ respectively.

Now consider the general case of circles with centers $A_{1}, A_{2}, A_{3}$ and radii $r_{1}, r_{2}, r_{3}$. Denote their radical center by

$$
P_{0}=\left[\delta_{1}, \delta_{2}, \delta_{3}\right]
$$

In what follows there is no loss of generality in assuming that $P_{0}$ is in the interior of $A_{1} A_{2} A_{3}$. Near $A_{1}$ we have

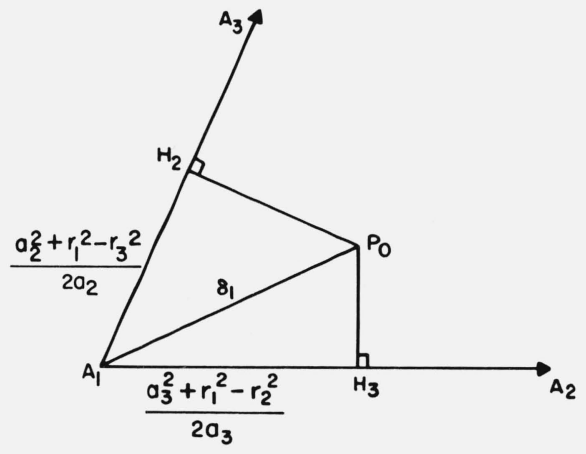


Let $\alpha_{1}$ denote the interior angle at $A_{1}$. Then $\left|H_{2}, H_{3}\right|=\delta_{1} \sin \alpha_{1} \cdot{ }^{3}$ The formula for $\cos \alpha_{1}$ in the triangle $\mathrm{A}_{1} \mathrm{H}_{2} \mathrm{H}_{3}$ is

(6) $\quad 2\left(\frac{a_{2}^{2}+r_{1}^{2}-r_{3}^{2}}{2 a_{2}}\right)\left(\frac{a_{3}^{2}+r_{1}^{2}-r_{2}^{2}}{2 a_{3}}\right) \cos \alpha_{1}=\left(\frac{a_{2}^{2}+r_{1}^{2}-r_{3}^{2}}{2 a_{2}}\right)^{2}+\left(\frac{a_{3}^{2}+r_{1}^{2}-r_{2}^{2}}{2 a_{3}}\right)^{2}-\left(\delta_{1} \sin \alpha_{1}\right)^{2}$.

In order to simplify this equation we shall need a few definitions and formulas. Define

$$
\begin{aligned}
& c_{1}=a_{2}^{2}+a_{3}^{2}-a_{1}^{2} \\
& c_{2}=a_{1}^{2}+a_{3}^{2}-a_{2}^{2} \\
& c_{3}=a_{1}^{2}+a_{2}^{2}-a_{3}^{2} .
\end{aligned}
$$

Note that

$$
c_{1}=2 a_{2}^{2}-c_{3}=2 a_{3}^{2}-c_{2}
$$

and that

(9)

$$
2 a_{2} a_{3} \cos \alpha_{1}=c_{1} \text {. }
$$

Since

$$
a_{2} a_{3} \sin \alpha_{1}=2 \Delta
$$

we have

$$
c_{1}^{2}+16 \Delta^{2}=4 a_{2}^{2} a_{3}^{2}
$$

Now return to eq (6). Multiply through by $4 a_{2}^{2} a_{3}^{2}$, and use eqs (7) through (11) to simplify. We get $16 \Delta^{2} f\left(r_{1}^{2}, r_{2}^{2}, r_{3}^{2}\right)=16 \Delta^{2}\left(r_{1}^{2}-\delta_{1}^{2}\right)$, where

$$
\begin{aligned}
16 \Delta^{2} f\left(x_{1}, x_{2}, x_{3}\right) & =\sum_{k=1}^{3} a_{k}^{2} c_{k} x_{k}-a_{1}^{2} a_{2}^{2} a_{3}^{2} \\
& -\frac{1}{2}\left\{c_{1}\left(x_{2}-x_{3}\right)^{2}+c_{2}\left(x_{1}-x_{3}\right)^{2}+c_{3}\left(x_{1}-x_{2}\right)^{2}\right\} .
\end{aligned}
$$

Generally:

$$
f\left(r_{1}^{2}, r_{2}^{2}, r_{3}^{2}\right)=r_{k}^{2}-\delta_{k}^{2} . \quad k=1,2,3 .
$$

If $f\left(r_{1}^{2}, r_{2}^{2}, r_{3}^{2}\right)=0$ then $r_{k}=\delta_{k}$ for all $k=1,2,3$ so that $P_{0}=\left[r_{1}, r_{2}, r_{3}\right]$.

Now notice that

$$
\sum_{k=1}^{3} a_{k}^{2} c_{k}=F\left(a_{1}^{2}, a_{2}^{2}, a_{3}^{2}\right)=16 \Delta^{2}
$$

\footnotetext{
${ }^{3}$ The circle with diameter $\overline{A_{1} P_{0}}$ goes through $H_{2}$ and $H_{3}$. Thus $\overline{H_{2} H_{3}}$ is a chord with opposite angle $\alpha_{1}$.
} 
so that

$$
f\left(x_{1}-t, x_{2}-t, x_{3}-t\right)=f\left(x_{1}, x_{2}, x_{3}\right)-t \quad \text { all } t .
$$

Let $x_{k}=r_{k}^{2}$ for all $k=1,2,3$ and let $t=f\left(r_{1}^{2}, r_{2}^{2}, r_{3}^{2}\right)$. Since (13) implies $x_{k}-t=\delta_{k}^{2}$ for all $k=1,2,3$, we have $f\left(\delta_{1}^{2}, \delta_{2}^{2}, \delta_{3}^{2}\right)=0$. As we pointed out before, any point $P=\left[d_{1}, d_{2}, d_{3}\right]$ can be a radical center. The above shows that $f\left(d_{1}^{2}, d_{2}^{2}, d_{3}^{2}\right)=0$.

Combining this with our remarks following eq (13), we have proved the first part of the following theorem:

TheOREM. Let $\mathrm{d}_{1}, \mathrm{~d}_{2}, \mathrm{~d}_{3}$ be any real nonnegative numbers. Then there is a point $\mathrm{P}=\left[\mathrm{d}_{1}, \mathrm{~d}_{2}, \mathrm{~d}_{3}\right]$ if and only if $\mathrm{f}\left(\mathrm{d}_{1}^{2}, \mathrm{~d}_{2}^{2}, \mathrm{~d}_{3}^{2}\right)=0$ ( $\mathrm{f}$ defined in (12)). In that case $\mathrm{P}=\left(\mathrm{f}_{1}\left(\mathrm{~d}_{1}^{2}, \mathrm{~d}_{2}^{2}, \mathrm{~d}_{3}^{2}\right), \mathrm{f}_{2}\left(\mathrm{~d}_{1}^{2}, \mathrm{~d}_{2}^{2}, \mathrm{~d}_{3}^{2}\right)\right.$, $\left.\mathrm{f}_{3}\left(\mathrm{~d}_{1}^{2}, \mathrm{~d}_{2}^{2}, \mathrm{~d}_{3}^{2}\right)\right)$ where $\mathrm{f}_{\mathrm{k}}\left(\mathrm{x}_{1}, \mathrm{x}_{2}, \mathrm{x}_{3}\right)=\partial \mathrm{f}\left(\mathrm{x}_{1}, \mathrm{x}_{2}, \mathrm{x}_{3}\right) / \partial \mathrm{x}_{\mathrm{k}}$ for all $\mathrm{k}=1,2,3$.

For clarity, we write out the $f_{k}$ :

$$
\begin{aligned}
& 16 \Delta^{2} f_{1}\left(x_{1}, x_{2}, x_{3}\right)=a_{1}^{2} c_{1}+c_{3}\left(x_{2}-x_{1}\right)+c_{2}\left(x_{3}-x_{1}\right) \\
& 16 \Delta^{2} f_{2}\left(x_{1}, x_{2}, x_{3}\right)=a_{2}^{2} c_{2}+c_{3}\left(x_{1}-x_{2}\right)+c_{1}\left(x_{3}-x_{2}\right) \\
& 16 \Delta^{2} f_{3}\left(x_{1}, x_{2}, x_{3}\right)=a_{3}^{2} c_{3}+c_{2}\left(x_{1}-x_{3}\right)+c_{1}\left(x_{2}-x_{3}\right) .
\end{aligned}
$$

Note that (14) implies

$$
f_{1}\left(x_{1}, x_{2}, x_{3}\right)+f_{2}\left(x_{1}, x_{2}, x_{3}\right)+f_{3}\left(x_{1}, x_{2}, x_{3}\right)=1 .
$$

Also note that

$$
f_{k}\left(x_{1}-t, x_{2}-t, x_{3}-t\right)=f_{k}\left(x_{1}, x_{2}, x_{3}\right) \quad \text { all } t ; k=1,2,3 .
$$

In the process of proving the last part of the theorem, we shall need the following computations. The locus of points with $b_{3}=1$ is a line through $A_{3}$ parallel to $A_{1} A_{2}$. Naturally if $P=\left(b_{1}, b_{2}, b_{3}\right)$ then $b_{1}+b_{2}=0$ (see (3)). Suppose $b_{1} \leqslant 0$.

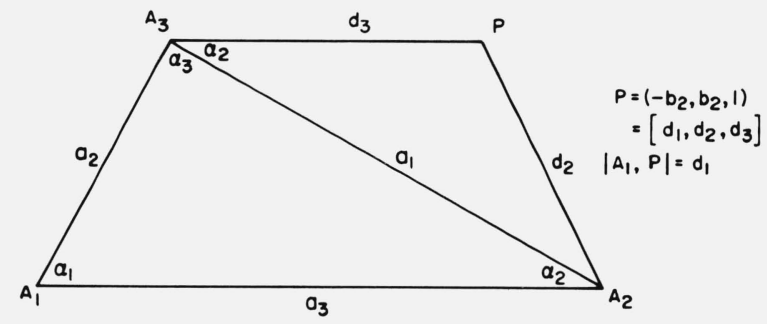

The area of $P A_{2} A_{3}$ is $\Delta \cdot\left|-b_{2}\right|$. It is also $\frac{1}{2} a_{1} d_{3} \sin \alpha_{2}$.

Therefore

$$
d_{3}=a_{3} b_{2}
$$

Using the cosine formula in that triangle yields

$$
\begin{aligned}
d_{2}^{2}= & d_{3}^{2}+a_{1}^{2}-2 a_{1} d_{3} \cos \alpha_{2} \\
& =a_{3}^{2} b_{2}^{2}+a_{2}^{2}-b_{2} c_{2} .
\end{aligned}
$$

Using the cosine formula in the triangle $A_{1} A_{3} P$ we have

$$
d_{1}^{2}=d_{3}^{2}+a_{2}^{2}-2 a_{2} d_{3} \cos \left(\alpha_{2}+\alpha_{3}\right)
$$




$$
=a_{3}^{2} b_{2}^{2}+a_{2}^{2}+b_{2} c_{1}
$$

Now use eq (18) with each $x_{k}=d_{k}^{2}$ and $t=d_{3}^{2}=a_{3}^{2} b_{2}^{2}$. For $k=1$ we have (using (20) and (21)):

$$
\begin{aligned}
16 \Delta^{2} f_{1}\left(d_{1}^{2}, d_{2}^{2}, d_{3}^{2}\right) & =16 \Delta^{2} f_{1}\left(a_{2}^{2}+b_{2} c_{1}, a_{1}^{2}-b_{2} c_{2}, 0\right) \\
& =a_{1}^{2} c_{1}+c_{3}\left(a_{1}^{2}-a_{2}^{2}-b_{2}\left(c_{1}+c_{2}\right)\right)-c_{2}\left(a_{2}^{2}+b_{2} c_{1}\right)
\end{aligned}
$$

(using (16)). Since $a_{1}^{2} c_{1}+c_{3}\left(a_{1}^{2}-a_{2}^{2}\right)=c_{2} a_{2}^{2}\left(\right.$ note $\left.c_{1}+c_{3}=2 a_{2}^{2}, c_{2}+c_{3}=2 a_{1}^{2}\right)$, and since

$$
c_{1} c_{2}+c_{1} c_{3}+c_{2} c_{3}=16 \Delta^{2},
$$

$\left(c_{1}+c_{2}=2 a_{3}^{2}\right.$, then add the three symmetric formulas, and use eq (14)), we have

$$
f_{1}\left(d_{1}^{2}, d_{2}^{2}, d_{3}^{2}\right)=-b_{2}=b_{1}
$$

as desired.

Similar computations prove $f_{2}\left(d_{1}^{2}, d_{2}^{2}, d_{3}^{2}\right)=b_{2}$ and (as a check), $f_{3}\left(d_{1}^{2}, d_{2}^{2}, d_{3}^{2}\right)=1=b_{3}$. The case $b_{2} \leqslant 0$ is handled similarly to prove the last part of the theorem for the case $b_{3}=1$.

Return to eq (12) and solve $f\left(x_{1}, x_{2}, x_{3}\right)=0$ for $x_{1}$. An intermediary stage is the equation

$$
16 \Delta^{2}\left\{f_{1}\left(x_{1}, x_{2}, x_{3}\right)\right\}^{2}=2 a_{1}^{2}\left(x_{2}+x_{3}\right)+2 x_{2} x_{3}-a_{1}^{4}-x_{2}^{2}-x_{3}^{2} .
$$

The r.h.s. is recognized to be $F\left(a_{1}^{2}, x_{2}, x_{3}\right)$. Let $x_{k}=d_{k}^{2}$ for all $k=1,2,3$, and use (4), to get $\left\{f_{1}\left(d_{1}^{2}\right.\right.$, $\left.\left.d_{2}^{2}, d_{3}^{2}\right)\right\}^{2}=b_{1}^{2}$. Generally

$$
f_{k}\left(d_{1}^{2}, d_{2}^{2}, d_{3}^{2}\right)= \pm b_{k} \quad k=1,2,3
$$

Set $f_{k}=f_{k}\left(d_{1}^{2}, d_{2}^{2}, d_{3}^{2}\right)$ for all $k=1,2,3$. Equations (3) and (17), showing $\Sigma b_{k}=\Sigma f_{k}=1$, imply that we cannot have $f_{k}=-b_{k}$ for all $k$. Suppose $f_{3}=b_{3}$. Then

$$
f_{1}+f_{2}=1-f_{3}=1-b_{3}=b_{1}+b_{2} .
$$

If $f_{1}=b_{1}$ then $f_{2}=b_{2}$, and conversely. The only open case is $f_{1}=-b_{1}, f_{2}=-b_{2}$. This implies $b_{1}+b_{2}=0$, whence $b_{3}=1$. We have already covered this case, so the proof of the theorem is complete.

An interesting implication for $P_{0}$ is immediate. Use eq (18) with $x_{k}=r_{k}^{2}$ for all $k=1,2,3$ and $t=f\left(r_{1}^{2}, r_{2}^{2}, r_{3}^{2}\right)$ as before. The result is $f_{k}\left(\delta_{1}^{2}, \delta_{2}^{2}, \delta_{3}^{2}\right)=f_{k}\left(r_{1}^{2}, r_{2}^{2}, r_{3}^{2}\right)$ for all $k=1,2,3$. In other words the area coordinates for the radical center of three circles with centers at $A_{1}, A_{2}, A_{3}$ and radii $r_{1}, r_{2}, r_{3}$ respectively are given by

$$
P_{0}=\left(f_{1}\left(r_{1}^{2}, r_{2}^{2}, r_{3}^{2}\right), f_{2}\left(r_{1}^{2}, r_{2}^{2}, r_{3}^{2}\right), f_{3}\left(r_{1}^{2}, r_{2}^{2}, r_{3}^{2}\right)\right) .
$$

Of course, the distance coordinates are given by

$$
\boldsymbol{P}_{0}=<r_{1}^{2}-f\left(r_{1}^{2}, r_{2}^{2}, r_{3}^{2}\right), r_{2}^{2}-f\left(r_{1}^{2}, r_{2}^{2}, r_{3}^{2}\right), r_{3}^{2}-f\left(r_{1}^{2}, r_{2}^{2}, r_{3}^{2}\right)>\text {. }
$$

If we wish to find the (0 to 8 ) circles simultaneously tangent to the three circles used above, ve can do so through $f$, to obtain four quadratic equations whose solutions solve the problem. The point is that a circle of radius $r$ which is simultaneously tangent to all three circles has a center 


$$
P=\left[r_{1}+\epsilon_{1} r, r_{2}+\epsilon_{2} r, r_{3}+\epsilon_{3} r\right]
$$

with each $\epsilon_{k}= \pm 1$ depending on whether the tangency is "outside" $(\epsilon=1)$ or "inside" $(\epsilon=-1)$. Simplifying

$$
f\left(\left(r_{1}+\epsilon_{1} r\right)^{2},\left(r_{2}+\epsilon_{2} r\right)^{2},\left(r_{3}+\epsilon_{3} r\right)^{2}\right)=0,
$$

we get a quadratic equation in $r$ (with constant term $\left.f\left(r_{1}^{2}, r_{2}^{2}, r_{3}^{2}\right)\right)$. If $r$ is a negative root of that equation, we simply "assign" $-r$ to $-\epsilon_{1},-\epsilon_{2},-\epsilon_{3}$ since $\epsilon_{k} r=\left(-\epsilon_{k}\right)(-r), k=1,2,3$.

Thus we can cover all solutions with just four triples of $\epsilon$ 's, no two of which are negatives of each other.

We end this note with a list of formulas connecting the area and distance coordinates of a point

$$
P=\left(b_{1}, b_{2}, b_{3}\right)=\left[d_{1}, d_{2}, d_{3}\right]
$$

The formulas are given without proof, but are easily derived, with extensive use of the formula for the distance between $P$ and $P^{\prime}=\left(b_{1}^{\prime}, b_{2}^{\prime}, b_{3}^{\prime}\right)$ :

$$
2\left|P, P^{\prime}\right|^{2}=\sum_{k=1}^{3} c_{k}\left(b_{k}-b_{k}^{\prime}\right)^{2}
$$

First we complete the connection between the coordinates, begun in the last formula of the theorem, with

$$
\begin{aligned}
& d_{1}^{2}=a_{3}^{2} b_{2}^{2}+c_{1} b_{2} b_{3}+a_{2}^{2} b_{3}^{2} \\
& d_{2}^{2}=a_{3}^{2} b_{1}^{2}+c_{2} b_{1} b_{3}+a_{1}^{2} b_{3}^{2} \\
& d_{3}^{2}=a_{2}^{2} b_{1}^{2}+c_{3} b_{1} b_{2}+a_{1}^{2} b_{2}^{2}
\end{aligned}
$$

or

$$
2 d_{k}^{2}=\left(1-2 b_{k}\right) c_{k}+\sum_{n=1}^{3} b_{n}^{2} c_{n} \quad k=1,2,3 .
$$

Let $R$ denote the circumradius of $A_{1} A_{2} A_{3}$, and $\rho_{P}$ the distance from $P$ to the circumcenter. The latter has area coordinates $a_{k}^{2} c_{k} / 16 \Delta^{2}, k=1,2,3$. Also $4 \Delta R=a_{1} a_{2} a_{3}$.

Define

$$
G_{P}=R^{2}-\rho_{P}^{2}
$$

Then $G_{P}$ can be found using only the $b_{k}$ :

$$
\begin{aligned}
G_{P} & =a_{1}^{2} b_{2} b_{3}+a_{2}^{2} b_{1} b_{3}+a_{3}^{2} b_{1} b_{2} \\
& =\frac{1}{2} \sum_{k=1}^{3}\left(b_{k}-b_{k}^{2}\right) c_{k} ;
\end{aligned}
$$

or only the $d_{k}$ :

$$
G_{P}=R^{2}-\left(c_{1}\left(d_{2}-d_{3}\right)^{2}+c_{2}\left(d_{1}-d_{3}\right)^{2}+c_{3}\left(d_{1}-d_{2}\right)^{2}\right) / 32 \Delta^{2}
$$




$$
\begin{aligned}
& =R^{2}-\left(a_{1}^{2}\left(d_{1}^{2}-d_{2}^{2}\right)\left(d_{1}^{2}-d_{3}^{2}\right)+a_{2}^{2}\left(d_{2}^{2}-d_{1}^{2}\right)\left(d_{2}^{2}-d_{3}^{2}\right)+a_{3}^{2}\left(d_{3}^{2}-d_{1}^{2}\right)\left(d_{3}^{2}-d_{2}^{2}\right)\right) / 16 \Delta^{2} \\
& =\left(2 a_{1}^{2} a_{2}^{2} a_{3}^{2}-\sum a_{k}^{2} c_{k} d_{k}^{2}\right) / 16 \Delta^{2} ;
\end{aligned}
$$

or symmetric combinations:

$$
\begin{aligned}
G_{P} & =\sum_{k=1}^{3} b_{k} d_{k}^{2} \\
& =\sum_{k=1}^{3}\left(a_{k}^{2}-2 d_{k}^{2}+b_{k}^{2} c_{k}\right) / 4 \\
& =\sum_{k=1}^{3}\left(a_{k}^{2}-2 d_{k}^{2}+b_{k} c_{k}\right) / 6 \\
& =\sum_{k=1}^{3}\left(a_{k}^{2}-d_{k}^{2}-b_{k} a_{k}^{2}\right) / 3 \\
& =2\left\{b_{1} a_{2}^{2} a_{3}^{2}+b_{2} a_{1}^{2} a_{3}^{2}+b_{3} a_{1}^{2} a_{2}^{2}-\sum_{k=1}^{3} a_{k}^{2} d_{k}^{2}\right\} / \sum_{k=1}^{3} a_{k}^{2} \\
& =\left\{b_{1} b_{2} b_{3} \sum_{k=1}^{3} a_{k}^{2}+\sum_{k=1}^{3} b_{k}^{2} d_{k}^{2}\right\} / 2\left(b_{1} b_{2}+b_{1} b_{3}+b_{2} b_{3}\right)
\end{aligned}
$$

(except at a vertex). Other relations are

$$
\begin{gathered}
d_{1}^{2}=b_{2} a_{3}^{2}+b_{3} a_{2}^{2}-G_{P} \quad \text { etc. } \\
b_{1} c_{1}=2 G_{P}-a_{1}^{2}+d_{2}^{2}+d_{3}^{2} \quad \text { etc. } \\
\left(1-b_{1}\right) G_{P}=b_{1} d_{1}^{2}+b_{2} b_{3} a_{1}^{2} \quad \text { etc. } \\
2 a_{2}^{2} a_{3}^{2} b_{1}=c_{1} G_{P}+a_{2}^{2} d_{2}^{2}+a_{3}^{2} d_{3}^{2}-a_{1}^{2} d_{1}^{2} \quad \text { etc. }
\end{gathered}
$$

The pedal triangle of $P$, which has side lengths $a_{k} d_{k} / 2 R, k=1,2,3$, has area $\Delta\left|G_{P}\right| / 4 R^{2}$; i.e.,

$$
\begin{aligned}
16 \Delta^{2} G_{P}^{2} & =F\left(a_{1}^{2} d_{1}^{2}, a_{2}^{2} d_{2}^{2}, a_{3}^{2} d_{3}^{2}\right) \\
& =4 a_{2}^{2} a_{3}^{2} d_{2}^{2} d_{3}^{2}-\left(a_{2}^{2} d_{2}^{2}+a_{3}^{2} d_{3}^{2}-a_{1}^{2} d_{1}^{2}\right)^{2} \quad \text { etc. }
\end{aligned}
$$

If $P$ does not lie on the triangle $A_{1} A_{2} A_{3}$ then the lines $A_{1} P, A_{2} P, A_{3} P$ intersect the circle of radius $\rho_{P}$, concentric with the circumcircle, in $P$ and in points $A_{1}^{\prime}, A_{2}^{\prime}, A_{3}^{\prime}$ respectively which have (opposite) side lengths $\lambda_{P}\left|b_{k}\right| d_{k}$ for $k=1,2,3 ; \lambda_{P}=4 \Delta \rho_{P} / d_{1} d_{2} d_{3}$. The area of $A_{1}^{\prime} A_{2}^{\prime} A_{3}^{\prime}$ is $\Delta \lambda_{P}^{2}\left|b_{1} b_{2} b_{3}\right|$. Thus

$$
16 \Delta^{2}\left(b_{1} b_{2} b_{3}\right)^{2}=F\left(b_{1}^{2} d_{1}^{2}, b_{2}^{2} d_{2}^{2}, b_{3}^{2} d_{3}^{2}\right) .
$$

Finally, suppose $d_{k}^{2}=g_{k}(t)$, a differentiable function of $t$, for $k=1,2,3$. The $b_{k}$ will also be differentiable functions of $t$, (by the last statement of the theorem), and $b_{k}^{\prime}$ will denote the derivative. We have 


$$
\sum_{k=1}^{3} b_{k} g_{k}^{\prime}(t)=0
$$

and

$$
g_{1}^{\prime}(t)+c_{1} b_{1}^{\prime}=g_{2}^{\prime}(t)+c_{2} b_{2}^{\prime}=g_{3}^{\prime}(t)+c_{3} b_{3}^{\prime}=\sum_{k=1}^{3} c_{k} b_{k} b_{k}^{\prime}
$$

(Paper 76B3\&4-367) 